Revista Arbitrada Interdisciplinaria KOINONIA

Año VI. Vol VI. N³. Edición Especial: Educación II. 2021

Hecho el depósito de Ley: FA2016000010 ISSN: 2542-3088

FUNDACIÓN KOINONIA (F.K). Santa Ana de Coro. Venezuela.

José Antonio Guaman-Duy; Sergio Constantino Ochoa-Encalada

http://dx.doi.org/10.35381/r.k.v6i3.1313

\title{
Educación, COVID-19 y familia en el proceso de aprendizaje en Educación General Básica
}

Education, COVID-19 and family in the learning process in Basic General Education

\author{
José Antonio Guaman-Duy \\ jose.guaman.67@est.ucacue.edu.ec \\ Universidad Católica de Cuenca, Azogues \\ Ecuador \\ https://orcid.org/0000-0003-3053-0486 \\ Sergio Constantino Ochoa-Encalada \\ scochoae@ucacue.edu.ec \\ Universidad Católica de Cuenca, Cuenca \\ Ecuador \\ https://orcid.org/0000-0003-3067-3719
}

Recepción: 10 de abril 2021

Revisado: 05 de mayo 2021

Aprobación: 30 de junio 2021

Publicación: 15 de julio 2021 
Revista Arbitrada Interdisciplinaria KOINONIA

Año VI. Vol VI. N³. Edición Especial: Educación II. 2021

Hecho el depósito de Ley: FA2016000010

ISSN: 2542-3088

FUNDACIÓN KOINONIA (F.K). Santa Ana de Coro. Venezuela.

José Antonio Guaman-Duy; Sergio Constantino Ochoa-Encalada

\title{
RESUMEN
}

El objetivo de la presente investigación es analizar el grado de involucramiento de la familia en el proceso de aprendizaje de los estudiantes en el contexto de la pandemia por COVID-19. Para ello se empleó una metodología descriptiva con un enfoque mixto, se aplicó una encuesta a una muestra de 77 padres de familia. Los resultados obtenidos demuestran que existe un grado de involucramiento aceptable de la familia en el proceso de aprendizaje, sin embargo, se identificaron ciertas limitantes que impiden un acompañamiento más efectivo, siendo la falta de tiempo, el desconocimiento de contenidos, así como del manejo de recursos tecnológicos los más significativos. Frente a esto, la propuesta es implementar un plan familiar de actuación efectiva que permitirá una gestión adecuada del tiempo en función de las necesidades, priorizando el control y acompañamiento a las actividades educativas en un ambiente armónico.

Descriptores: Proceso de aprendizaje; relación padres-escuela; familia; pandemia. (Palabras tomadas del Tesauro UNESCO).

\begin{abstract}
The objective of this research is to analyze the degree of family involvement in the learning process of students in the context of the COVID-19 pandemic. For this, a descriptive methodology with a mixed approach was used, a survey was applied to a sample of 77 parents. The results obtained show that there is an acceptable degree of involvement of the family in the learning process, however, certain limitations were identified that prevent a more effective accompaniment, being lack of time, ignorance of contents, as well as the management of technological resources the most significant. Faced with this, the proposal is to implement an effective family plan of action that will allow adequate time management based on needs, prioritizing control and monitoring of educational activities in a harmonious environment.
\end{abstract}

Descriptors: Learning processes; parent school relationship; family; pandemics. (Words taken from the UNESCO Thesaurus). 


\section{INTRODUCCIÓN}

La actual pandemia provocada por el COVID-19 y las necesarias medidas de confinamiento obligatorio para controlar su propagación, plantean retos sin precedentes para los sistemas educativos a nivel mundial. El cierre generalizado de miles de centros educativos ha suspendido la interacción presencial del estudiante con el docente, proceso en el que se produce la transmisión y adquisición de conocimientos, habilidades y destrezas profundas, relevantes y significativas para todos los estudiantes.

En este proceso, el rol del docente como acompañante es crucial, puesto que orienta el aprendizaje mediante estrategias activas y participativas. Sin embargo, en el contexto de la virtualidad que hoy estamos atravesando, esta función de guiar el aprendizaje se vuelve mucho más compleja debido a múltiples factores como disponibilidad de recursos tecnológicos, acceso a internet, características socio emocionales individuales de cada estudiante, características socioculturales de los hogares, etc. Bajo esta perspectiva, los padres de familia son quienes deben asumir el acompañamiento en el proceso educativo de los estudiantes.

Pocas veces la humanidad ha enfrentado crisis tan graves y complejas como la provocada por la pandemia actual causada por la aparición de un nuevo tipo de coronavirus conocido como SARS-COV 2. Dicha crisis global ha afectado todos los ámbitos de la sociedad, pero sin duda alguna el sistema educativo ha sido el que ha debido afrontar los retos más serios debido a la suspensión masiva de actividades educativas presenciales. De acuerdo con el informe presentado por la (CEPAL-UNESCO, 2020), para mediados de mayo de 2020 más de 1200 millones de estudiantes de todos los niveles dejaron de tener contacto presencial con sus maestros. En América Latina y el Caribe más de 160 millones de estudiantes se acogieron obligatoriamente al trabajo en casa.

En nuestro país, el 16 de marzo de 2020 se declara el estado de excepción por calamidad pública mediante decreto ejecutivo No. 1017. En concordancia con este documento legal, el Ministerio de Educación, mediante Acuerdo Ministerial del 3 de abril del mismo año 


\footnotetext{
Revista Arbitrada Interdisciplinaria KOINONIA

Año VI. Vol VI. N`3. Edición Especial: Educación II. 2021

Hecho el depósito de Ley: FA2016000010

ISSN: 2542-3088

FUNDACIÓN KOINONIA (F.K). Santa Ana de Coro. Venezuela.

ratifica la suspensión de clases en todo el territorio nacional, para todas las instituciones educativas, tanto públicas, como fiscomisionales y particulares en todas sus modalidades. Así mismo dispone que todas las instituciones educativas continuarán con el cumplimiento del cronograma escolar en la modalidad de teletrabajo (Ministerio de Educación, 2020a).

Frente a esta inusitada situación las familias ecuatorianas se han visto en la obligación de buscar los medios para tratar de cumplir adecuadamente el rol que le corresponde como acompañante del proceso educativo de sus hijos en cada uno de los hogares. Todo esto en un escenario adverso, puesto que en nuestro país solo el 37 por ciento de los hogares tiene acceso a internet, esto significa que alrededor de 6 de cada 10 niños no tienen la posibilidad de continuar sus estudios a través de plataformas digitales. La situación es mucho más grave para los niños de zonas rurales, en donde solo el 16 por ciento de los hogares tiene este servicio (Vohlonen, 2020).

No obstante, según cifras de (Instituto Nacional de Estadisticas y Censo INEC, 2018), en el país el $50 \%$ de los hogares del área urbana sí cuenta con un teléfono inteligente, pero, solo $23 \%$ de las familias del área rural cuenta con este dispositivo que a partir de ahora será esencial para lograr que la educación no se detenga debido a la emergencia sanitaria. Esta situación supone un desafío y a la vez una oportunidad para todos los actores del sistema educativo para repensar la educación y plantear propuestas y nuevas formas de enseñar y aprender. En este contexto, sin duda alguna el reto más difícil es para la familia, que debe adaptarse a esta nueva modalidad de aprendizaje.

Si bien las familias, son agentes de primer orden y fuente de aprendizaje que complementan el proceso formativo del estudiante y constituyen un eslabón imprescindible en el logro de las metas educativas que pueden ser fortalecidas por diversos campos de actuación como acompañamiento y revisión de tareas escolares, promoción de actividades extracurriculares, tareas domésticas, entre otras. (MuñozMoreno \& Lluch-Molins, 2020). Sin embargo, en el contexto de la pandemia el rol de la familia en la continuidad del estudio es preocupante, puesto que la desigualdad 
socioeconómica de nuestro entorno supone serias limitaciones de acceso a recursos tecnológicos, y demás materiales escolares necesarios para un correcto aprendizaje. La Unidad Educativa Comunitaria Intercultural Bilingüe (UECIB) Suscal, es una Institución fiscal que se encuentra ubicada en el área rural del cantón Suscal, misma que desde el inicio de la pandemia ha debido enfrentar una seria problemática debido a que un porcentaje importante de estudiantes no cuentan con recursos tecnológicos ni con acceso a Internet, por lo tanto, su proceso formativo se ve limitado. Específicamente en el subnivel de Educación General Básica Superior, se ha evidenciado que las familias tienen varias dificultades en el proceso de acompañamiento a la labor educativa del estudiante en casa, debido a sus condiciones socioculturales y económicas.

La mayoría de padres de familia al ser del área rural, salen a sus labores agrícolas durante el día por lo tanto disponen de muy poco tiempo para dedicar al control de tareas. En este marco, el problema del presente estudio de investigación es conocer ¿Cuál es el rol de la familia en el proceso de aprendizaje de los estudiantes de Básica Superior de la UECIB "Suscal" en el contexto de la pandemia por COVID-19?

Considerando la situación anteriormente descrita en la que debido a la limitación de recursos tecnológicos los estudiantes reciben sus guías de aprendizaje dosificadas, así como los demás apoyos pedagógicos preparados por los docentes mediante un grupo de WhatsApp, podremos comprender los grandes desafíos que los hogares deben superar en el acompañamiento de los educandos.

Por lo tanto, el objetivo planteado es analizar el grado de involucramiento de la familia en el proceso de aprendizaje de los estudiantes de Educación General Básica Superior de la UECIB "Suscal" en el contexto de la pandemia por COVID-19. De esta manera, en base a los resultados será posible tomar mejores decisiones que permitan efectivizar la actuación de la familia y, por ende, mejorar desempeño escolar de todos los estudiantes.

\section{Referencial teórico}

Diversos estudios han evidenciado la importancia trascendental que tiene la actuación de la familia como complemento en el proceso de enseñanza-aprendizaje de los estudiantes. 
En el hogar es donde se cimientan y fortalecen las bases de la personalidad del individuo, sus valores, principios y su capacidad para afrontar los desafíos que plantea la sociedad, precisamente uno de los primeros desafíos a afrontar por los niños es su escolarización. A este respecto (Pire-Rojas, 2020), comenta que en el ámbito educativo la efectiva participación de los padres es fundamental, puesto que se constituye en un medio de apoyo al logro de los objetivos educativos, complementando y reforzando los conocimientos que han sido impartidos en el centro escolar. Por lo visto, es importante que entre la escuela y la familia haya una interacción proactiva constante, sustentada en el compromiso que cada agente del proceso educativo asume para mejorar la adquisición de conocimientos, habilidades y destrezas.

De acuerdo con (Méndez \& Jaimes, 2018), la interacción familiar desde edades tempranas influye en las diferentes etapas de la vida de las personas, facilitando o dificultando los procesos formativos. Se entiende entonces, que el rol de la familia es importante en el desarrollo cognitivo, puesto que, de una $u$ otra forma influye en el rendimiento académico de los estudiantes.

Con esta premisa podemos inferir que en un hogar donde no hay una relación familiar saludable, donde los padres se desentienden de su compromiso con la educación de sus hijos, o donde haya escasez de recursos o una deficiencia del capital cultural, sin duda el estudiante no encontrará apoyo en su entorno familiar para reforzar sus aprendizajes, por lo tanto, está en desventaja frente a otros, cuya familia si consideren la importancia de su participación activa en la educación.

Por otro lado, esta suspensión de clases presenciales debido a la pandemia ha delegado a las familias mucha más responsabilidad de atención a sus hijos en el hogar. Las diferentes plataformas educativas, lecciones online, recursos digitales, aplicaciones móviles, o los grupos estudio por WhatsApp, demandan necesariamente la participación de los familiares, mucho más en edades tempranas que requieren de mayor control del trabajo autónomo del estudiante frente a las pantallas. Sin embargo, es preocupante el hecho de que existen familias que no disponen de un computador en sus casas, o que 
todos sus miembros deben compartir un solo aparato electrónico o familias que ni siquiera cuentan con conexión a Internet (Muñoz-Moreno \& Lluch-Molins, 2020).

Al trasladarse el proceso educativo a los hogares, se han hecho aún más evidentes las brechas educativas, debido a que los hogares que no disponen de los medios necesarios para brindar apoyo al proceso educativo del educando (Formichella \& Krüger, 2020). En tal escenario, es muy difícil que se pueda aprovechar adecuadamente las estrategias alternativas que se han implementado por parte de los docentes, estrategias que, dicho sea de paso, no podrá suplantar al contacto directo entre profesor y alumno. En este marco de escasez, las condiciones socioeconómicas, así como el nivel de instrucción de los padres, se hace más relevante, problemática que se profundiza cuando los hogares son del área rural, donde en muchas ocasiones ni siquiera hay cobertura celular. Es entendible además que los padres deban salir a realizar sus labores agrícolas durante el día y no dispongan del tiempo suficiente para acompañar a sus hijos.

La pandemia por COVID-19 ha causado una seria crisis sin precedentes en todos los ámbitos de la sociedad. En el campo educativo esta emergencia sanitaria ha dado lugar al cierre masivo e imprevisto de miles de centros educativos a escala global. Ante esta situación los sistemas educativos se han visto en la obligación de tomar medidas generales como el surgimiento de modalidades de aprendizaje a distancia, mediante la utilización de una diversidad de formatos y plataformas (CEPAL-UNESCO, 2020). En este nuevo contexto para el que ni docentes ni estudiantes estaban preparados, la familia ha debido forzosamente asumir un rol protagónico en el acompañamiento de los estudiantes en el seno de los hogares.

Sin embargo, considerando la desigualdad en el acceso al Internet y demás recursos tecnológicos, que especialmente afecta a los sectores más vulnerables, se ha priorizado los esfuerzos por mantener el contacto y a fortalecer las condiciones socioemocionales de los estudiantes. En este marco, en el Ecuador, el (Ministerio de Educación, 2020b), se ha propuesto mantener la continuidad del proceso educativo, guiando la labor docente 
principalmente hacia la contención emocional, al desarrollo de los aprendizajes y la atención a la diversidad.

Para el logro de este objetivo se ha optado por la implementación de un currículo priorizado para la emergencia, mismo que está contextualizado a la realidad del país y a los desafíos planteados por la emergencia con un enfoque interdisciplinar. Con lo que se busca hacer más llevadero el proceso educativo priorizando los objetivos de aprendizaje y los contenidos esenciales, promoviendo el desarrollo de habilidades para la vida, valores, $y$, fortaleciendo el apoyo emocional.

El proceso de aprendizaje es de naturaleza extremadamente compleja, su esencia es la adquisición de un nuevo conocimiento, habilidad o capacidad. Para que dicho proceso pueda ser considerado como aprendizaje efectivo, debe tener la capacidad de perdurar en el tiempo y más que nada, contribuir a la solución de problemas reales del individuo (Alfonso, 2003). El proceso de enseñanza aprendizaje se considera como un espacio donde el principal protagonista es el estudiante y el docente cumple con una función de facilitador y mediador de los procesos de aprendizaje. En el actual contexto, la familia se convierte en un apoyo fundamental al rol docente en el hogar.

Como ya se puntualizó, el rol familiar en el proceso de aprendizaje es de vital importancia, puesto que es el primer medio de socialización del individuo, en este medio es donde se transmiten los primeros patrones culturales, los primeros responsables de la educación de los hijos son los padres. Las familias con la escuela comparten la labor de formar, de educar, complementando y fortaleciendo sus experiencias de aprendizaje.

Para lograr el objetivo de formar integralmente a la persona es necesario una unidad de criterios educativos en casa y en la escuela. Obviamente, para conseguir ese fin se requiere de una comunicación y coordinación permanentes entre maestros y padres (Razeto-Pavez, 2016). Si por algún motivo falta la intervención de la familia, la educación de un niño es meramente académica, carece del factor emocional que precisamente se fomenta en la interacción social. 
En el actual contexto de confinamiento, en el que la escuela se ha trasladado al hogar, el sistema educativo ecuatoriano ha implementado el plan educativo denominado "aprendamos juntos en casa", con el objeto de precautelar la salud de los estudiantes en los tres niveles que lo conforman. En este sentido se establecen los roles de las familias en los siguientes aspectos: establecer mecanismos de comunicación con los docentes mediante cualquier medio tecnológico y estar atentos a sus orientaciones; en caso de tener acceso a Internet, hacer uso de la plataforma habilitada para el proceso; apoyar en el desarrollo de las actividades pedagógicas; y, mantenerse pendiente de la situación emocional de los estudiantes. Sin duda alguna, es un gran desafío que la familia tiene frente a la formación de sus hijos. Lo preocupante es que no todos los hogares se encuentran en las mismas condiciones para asumir tal responsabilidad (Ministerio de Educación, 2020b).

Por lo visto, parece que la igualdad de derechos una vez más demuestra ser una falacia. Si bien las familias están llamadas a colaborar con la educación, apoyando el aprendizaje del estudiantado, guiando en la realización de las tareas para dar continuidad al proceso educativo y así garantizar el Derecho a la Educación y la consecución de las metas educativas. No obstante, durante el distanciamiento social se hicieron más evidentes las grandes diferencias sociales. Mientras unos grupos de la sociedad dedican tiempo de calidad a sus hijos y profundizan los aprendizajes, otros grupos sociales, o bien no están en casa porque están trabajando, o no disponen de los medios, ni recursos, ni capacidades para convertirse en profesores sustitutos. Con esto, siempre habrá un grupo de estudiantes que están en desventaja (Muñoz-Moreno \& Lluch-Molins, 2020).

Más allá de las grandes limitaciones que existen, el rol de la familia es incuestionable, el trabajo colaborativo se convierte en un mecanismo esencial para la práctica educativa, en tal virtud los centros educativos tienen la obligación de estimular la implicación de los miembros de la familia, manteniendo una estrecha comunicación y coordinación. Más aún, en estos tiempos es crucial la empatía para comprender la realidad individual de cada hogar y de esa forma efectivizar estrategias conjuntas que permitan aportar 
positivamente al rendimiento escolar del estudiante fortaleciendo su capacidad de aprender a aprender.

Otro aspecto relevante en el proceso educativo es el aprendizaje autónomo, comprendido como un proceso en el que el estudiante autorregula su aprendizaje y toma conciencia de sus propios procesos cognitivos y socio-afectivos (Torrano et al. 2017). Este tipo de aprendizaje es hacia donde se debería enfocar todos los esfuerzos durante esta situación de virtualidad educativa de modo que el estudiante sea capaz de desarrollar destrezas para autorregular sus acciones para aprender y alcanzar las metas esperadas. Así pues, en el difuso panorama que atravesamos, será primordial que cada estudiante pueda organizar su tiempo y sus prioridades, planificar tareas y reflexionar sobre ellas.

En este sentido, (Enríquez \& Hernández, 2021), concluyen que en el contexto provocado por el COVID-19, la dimensión afectiva-emocional del estudiantado requiere de la participación activa del docente, y más aún de la familia, puesto que la presente emergencia pone en evidencia la gran necesidad de acompañamiento y escucha. Para lo cual es imperativo proponer experiencias de aprendizaje centradas en las necesidades, características e intereses de quien aprende.

\section{MÉTODO}

La presente investigación es de tipo no experimental, tiene un enfoque metodológico cuantitativo que de acuerdo con (Hernández et al. 2014), emplea la recolección de datos con el fin de probar hipótesis, basándose en la medición numérica y el análisis estadístico, con el objetivo de establecer pautas de comportamiento y probar teorías. La población para esta investigación fue de 120 padres de familia de Educación General Básica Superior, de la Unidad Educativa Comunitaria Intercultural Bilingüe "Suscal", ubicada en el cantón Suscal, provincia de Cañar, de donde se seleccionó mediante un muestreo aleatorio simple, una muestra de 77 representantes, considerando que cada elemento del universo objetivo y a cada posible muestra de un determinado tamaño, tienen la misma posibilidad de ser elegidos. 
Para lo cual se aplicó un nivel de confianza del $85 \%$ y un margen de error del $5 \%$. Para la recolección de datos se aplicó la técnica de la encuesta mediante un cuestionario estructurado con 10 preguntas, dirigido a padres de familia. Fue aplicado mediante un Formulario de Microsoft Forms, cuyo Link fue compartido en los grupos de WhatsApp, donde están agregados los representantes de los seis paralelos de Básica Superior.

Para el análisis de datos cuantitativos se utilizó el Software IBM SPSS Statistics, así como para la respectiva validación de la encuesta, alcanzando un coeficiente de fiabilidad Alpha de Cronbach de 0,701 . Los datos obtenidos fueron analizados mediante la estadística descriptiva con un $95 \%$ de coeficiente de confianza, en base a la prueba de normalidad de Kolmogorov-Smirnov, los resultados evidencian que todas las variables son de tipo paramétrico, o sea que su Sig. Bilateral es menor que 0,05.

\section{RESULTADOS}

Las variables a continuación analizadas permiten responder al objetivo de investigación que fue analizar el grado de involucramiento de la familia en el proceso de aprendizaje de los estudiantes de Educación Básica Superior, cuyos resultados se muestran a continuación:

\section{Tabla 1.}

Relación entre nivel educativo de los representantes y frecuencia de acompañamiento a las tareas escolares.

\begin{tabular}{|c|c|c|c|c|c|c|c|}
\hline & & \multicolumn{5}{|c|}{ Frecuencia de acompañamiento a las tareas escolares } & \multirow{2}{*}{ Total } \\
\hline & & Nunca & $\begin{array}{c}\text { Casi } \\
\text { nunca }\end{array}$ & $\begin{array}{l}\text { Algunas } \\
\text { veces }\end{array}$ & $\begin{array}{c}\text { Casi } \\
\text { siempre }\end{array}$ & Siempre & \\
\hline \multirow{4}{*}{$\begin{array}{l}\text { Causas de la } \\
\text { falta de } \\
\text { acompañamiento }\end{array}$} & Falta de tiempo & 0 & 1 & 9 & 8 & 7 & 25 \\
\hline & Falta de conocimientos & 1 & 7 & 7 & 5 & 3 & 23 \\
\hline & $\begin{array}{l}\text { No requiere } \\
\text { acompañamiento }\end{array}$ & 0 & 0 & 1 & 5 & 14 & 20 \\
\hline & $\begin{array}{l}\text { Desconocimiento del } \\
\text { manejo de recursos } \\
\text { tecnológicos }\end{array}$ & 0 & 0 & 0 & 5 & 4 & 9 \\
\hline Total & & 1 & 8 & 17 & 23 & 28 & 77 \\
\hline
\end{tabular}


Revista Arbitrada Interdisciplinaria KOINONIA

Año VI. Vol VI. N³. Edición Especial: Educación II. 2021

Hecho el depósito de Ley: FA2016000010 ISSN: 2542-3088

FUNDACIÓN KOINONIA (F.K). Santa Ana de Coro. Venezuela.

\begin{tabular}{|c|c|c|c|c|c|c|c|}
\hline & & \multicolumn{5}{|c|}{ Frecuencia de acompañamiento a las tareas escolares } & \multirow{2}{*}{ Total } \\
\hline & & Nunca & $\begin{array}{c}\text { Casi } \\
\text { nunca }\end{array}$ & $\begin{array}{l}\text { Algunas } \\
\text { veces }\end{array}$ & $\begin{array}{c}\text { Casi } \\
\text { siempre }\end{array}$ & Siempre & \\
\hline \multirow{5}{*}{$\begin{array}{l}\text { Causas de la } \\
\text { falta de } \\
\text { acompañamiento }\end{array}$} & Falta de tiempo & 0 & 1 & 9 & 8 & 7 & 25 \\
\hline & Falta de conocimientos & 1 & 7 & 7 & 5 & 3 & 23 \\
\hline & $\begin{array}{l}\text { No requiere } \\
\text { acompañamiento }\end{array}$ & 0 & 0 & 1 & 5 & 14 & 20 \\
\hline & $\begin{array}{l}\text { Desconocimiento del } \\
\text { manejo de recursos } \\
\text { tecnológicos }\end{array}$ & 0 & 0 & 0 & 5 & 4 & 9 \\
\hline & & \multicolumn{2}{|c|}{ Valor } & gl & \multicolumn{3}{|c|}{ Sig. asintótica (bilateral) } \\
\hline \multicolumn{2}{|c|}{ Chi-cuadrado de Pearson } & \multicolumn{2}{|c|}{$35,788^{a}$} & 12 & \multicolumn{3}{|c|}{0,000} \\
\hline \multicolumn{2}{|c|}{ Razón de verosimilitudes } & \multicolumn{2}{|c|}{38,362} & 12 & \multicolumn{3}{|c|}{0,000} \\
\hline \multicolumn{2}{|c|}{ Asociación lineal por lineal } & \multicolumn{2}{|c|}{7,863} & 1 & \multicolumn{3}{|c|}{0,005} \\
\hline \multicolumn{2}{|c|}{ N de casos válidos } & 77 & & & & & \\
\hline
\end{tabular}

a. 12 casillas $(60,0 \%)$ tienen una frecuencia esperada inferior a 5 . La frecuencia mínima esperada es ,12.

Fuente: Cuestionario.

La tabla 1 evidencia que más de la tercera parte de los padres de familia opinan que siempre acompañan las tareas escolares de sus hijos, de estos, la mitad responden que su representado no necesita acompañamiento, la otra cuarta parte responde que la causa de la falta de acompañamiento es la falta de tiempo, otra cantidad menor de encuestados afirma que la falta de acompañamiento es por desconocimiento del manejo de recursos tecnológicos, finalmente una mínima cantidad dicen que es por la falta de conocimientos. También se evidencia que casi la tercera parte de encuestados afirma que casi siempre acompaña la realización de tareas, de este grupo, la tercera parte dice que es por la falta de tiempo y los demás, en iguales proporciones responden que las causas de la falta de acompañamiento son las otras opciones planteadas.

Se observa también que un considerable número de representantes manifiesta que acompaña las tareas escolares algunas veces, de ellos la mayoría considera que la causa es la falta de tiempo, seguido por un número menor que dice que es por la falta de conocimientos. Por otro lado, hay un pequeño grupo que dice que casi nunca y nunca 
acompaña las actividades escolares, siendo la mayor causa la falta de conocimientos. En base al análisis de Chi-cuadrado, con un valor Sig. Bilateral de 0,000, es evidentemente menor a 0,05 , con lo que se asume una hipótesis de investigación, demostrando que existe relación entre la frecuencia de acompañamiento a las tareas escolares, y, las causas de la falta de acompañamiento.

\section{Tabla 2.}

Comunicación del padre de familia con el docente.

\begin{tabular}{llcccc}
\hline & Frecuencia & Porcentaje & Porcentaje válido & $\begin{array}{c}\text { Porcentaje } \\
\text { acumulado }\end{array}$ \\
\hline \multirow{4}{*}{ Válidos } & Nunca & 3 & 3,9 & 3,9 & 3,9 \\
& Casi nunca & 12 & 15,6 & 15,6 & 19,5 \\
& Algunas veces & 22 & 28,6 & 28,6 & 48,1 \\
& Casi siempre & 16 & 20,8 & 20,8 & 68,8 \\
& Siempre & 24 & 31,2 & 31,2 & 100,0 \\
\cline { 2 - 5 } & Total & $\mathbf{7 7}$ & $\mathbf{1 0 0 , 0}$ & $\mathbf{1 0 0 , 0}$ & \\
\hline
\end{tabular}

Fuente: Cuestionario.

En la tabla 2 se puede evidenciar que un $52 \%$ de padres de familia siempre y casi siempre se comunican con los docentes, para conocer el desempeño académico de los estudiantes, demostrando que, si hay interés por apoyar el proceso educativo en los hogares, sin embargo, también existe, aunque en menor cantidad, quienes casi nunca se ponen en contacto con el profesor, siendo un indicador de la deficiencia en el rol que la familia debe cumplir en el contexto de la virtualidad. 
Revista Arbitrada Interdisciplinaria KOINONIA

Año VI. Vol VI. N³. Edición Especial: Educación II. 2021

Hecho el depósito de Ley: FA2016000010 ISSN: 2542-3088

FUNDACIÓN KOINONIA (F.K). Santa Ana de Coro. Venezuela.

José Antonio Guaman-Duy; Sergio Constantino Ochoa-Encalada

\section{Tabla 3.}

Relación entre nivel educativo de los padres de familia y grado de comprensión de las tareas escolares.

\begin{tabular}{|c|c|c|c|c|c|c|c|}
\hline & & \multicolumn{5}{|c|}{ Grado de comprensión de las tareas escolares } & \multirow{2}{*}{ Total } \\
\hline & & Nada & Casi nada & Algo & Bastante & Mucho & \\
\hline \multirow{5}{*}{$\begin{array}{l}\text { Nivel educativo de } \\
\text { los padres de } \\
\text { familia }\end{array}$} & Ninguno & 10 & 3 & 12 & 2 & 3 & 30 \\
\hline & Primaria & 1 & 3 & 16 & 2 & 4 & 26 \\
\hline & Primaria incompleta & 1 & 3 & 3 & 0 & 1 & 8 \\
\hline & Secundaria & 0 & 0 & 1 & 1 & 5 & 7 \\
\hline & Superior & 0 & 0 & 2 & 1 & 3 & 6 \\
\hline \multicolumn{2}{|c|}{ Total } & 12 & 9 & 34 & 6 & 16 & 77 \\
\hline \multicolumn{8}{|c|}{ Pruebas de chi-cuadrado } \\
\hline & & \multicolumn{2}{|r|}{ Valor } & gl & \multicolumn{3}{|c|}{ Sig. asintótica (bilateral) } \\
\hline & \multicolumn{2}{|c|}{$35,212^{\mathrm{a}}$} & 16 & \multicolumn{3}{|c|}{0,004} \\
\hline \multirow{2}{*}{\multicolumn{2}{|c|}{$\begin{array}{l}\text { Razón de verosimilitudes } \\
\text { Asociación lineal por lineal }\end{array}$}} & \multicolumn{2}{|c|}{33,699} & 16 & \multicolumn{3}{|c|}{0,006} \\
\hline & & \multicolumn{2}{|c|}{14,931} & 1 & \multicolumn{3}{|c|}{0,000} \\
\hline \multicolumn{2}{|l|}{$\mathrm{N}$ de casos válidos } & \multicolumn{2}{|r|}{77} & & & & \\
\hline
\end{tabular}

Fuente: Cuestionario.

La tabla 3 muestra que casi la mitad de encuestados asumen que comprenden algo las tareas escolares, de este grupo, más de la mitad tiene educación primaria, otro porcentaje importante no tiene ningún nivel de educación, y otro grupo pequeño tiene primaria incompleta. Se puede ver también que casi la cuarta parte de representantes afirma que comprenden mucho las tareas escolares, siendo la mayoría quienes tienen educación secundaria, seguido por quienes tienen la primaria.

Luego se observa que un número importante dice no comprender nada y casi nada las tareas escolares, siendo la mayoría quienes no tienen ningún nivel académico. En base al análisis de Chi-cuadrado, con un valor Sig. Bilateral de 0,004, notablemente menor a 0,05 , con lo que se asume una hipótesis de investigación, demostrando que existe relación entre el nivel educativo de los padres de familia y el grado de comprensión de las tareas escolares. 
Revista Arbitrada Interdisciplinaria KOINONIA

Año VI. Vol VI. N³. Edición Especial: Educación II. 2021

Hecho el depósito de Ley: FA2016000010 ISSN: 2542-3088

FUNDACIÓN KOINONIA (F.K). Santa Ana de Coro. Venezuela.

José Antonio Guaman-Duy; Sergio Constantino Ochoa-Encalada

\section{Tabla 4.}

Relación entre disponibilidad de un medio de conectividad y disponibilidad de recursos para recibir clases y realizar tareas.

\begin{tabular}{|c|c|c|c|c|c|}
\hline & & \multicolumn{3}{|c|}{$\begin{array}{l}\text { Disponibilidad de recursos para recibir clases y } \\
\text { realizar tareas }\end{array}$} & \multirow{2}{*}{ Total } \\
\hline & & Computador & $\begin{array}{l}\text { Teléfono } \\
\text { inteligente }\end{array}$ & $\begin{array}{l}\text { Guías de trabajo } \\
\text { impresas }\end{array}$ & \\
\hline \multirow{4}{*}{$\begin{array}{l}\text { Disponibilidad } \\
\text { de un medio de } \\
\text { conectividad }\end{array}$} & Internet fijo & 4 & 40 & 0 & 44 \\
\hline & Datos móviles & 0 & 26 & 0 & 26 \\
\hline & Wifi de un vecino o familiar & 0 & 5 & 0 & 5 \\
\hline & Ninguno & 0 & 0 & 2 & 2 \\
\hline & Total & 4 & 71 & 2 & 77 \\
\hline \multicolumn{6}{|c|}{ Pruebas de chi-cuadrado } \\
\hline & & Valor & gl & \multicolumn{2}{|c|}{ Sig. asintótica (bilateral) } \\
\hline Chi-cuadrado d & e Pearson & $80,056^{a}$ & 6 & \multicolumn{2}{|c|}{0,000} \\
\hline Razón de veros & imilitudes & 22,975 & 6 & \multicolumn{2}{|c|}{0,001} \\
\hline Asociación linea & al por lineal & 8,430 & 1 & \multicolumn{2}{|c|}{0,004} \\
\hline \multicolumn{2}{|c|}{$\mathrm{N}$ de casos válidos } & 77 & & & \\
\hline
\end{tabular}

a. 10 casillas $(83,3 \%)$ tienen una frecuencia esperada inferior a 5 . La frecuencia mínima esperada es, 05.

Fuente: Cuestionario.

La tabla 4 demuestra que en la gran mayoría de hogares se dispone de un teléfono inteligente para recibir clases y realizar las tareas escolares, de ellos más de la mitad cuentan con servicio de Internet fijo, una cantidad menor tiene conectividad mediante datos móviles y pequeña cantidad utiliza el Wifi de un vecino o familiar. Por otro lado, un pequeño número de hogares tiene un computador con acceso al servicio de internet fijo, mientras que una mínima cantidad de familias no cuenta con ningún recurso tecnológico por lo que recibe guías de trabajo impresas para desarrollar el aprendizaje. En cuanto al análisis de Chi-cuadrado, con un valor Sig. Bilateral de 0,000, claramente menor a 0,05, con lo que se asume una hipótesis de investigación, demostrando que existe relación entre la disponibilidad de un medio de conectividad y la disponibilidad de recursos para recibir clases y realizar tareas. 
Los resultados obtenidos en la presente investigación muestran que, si bien los padres de familia están comprometidos con el apoyo al proceso formativo de sus hijos en casa, existen ciertas limitantes que impiden una actuación efectiva frente al rol fundamental que las actuales condiciones exigen de parte de la familia. En ese sentido, aspectos como la falta de tiempo, el desconocimiento básico de los contenidos y del manejo de recursos tecnológicos son las principales causas de la falta de acompañamiento a las tareas educativas, antes que el desinterés. Coincidiendo con esta situación, (Oliveira, 2021), concluye que la unión entre la escuela y la familia es fundamental, sin embargo, hay muchas familias que no pueden realizar el seguimiento necesario, ya sea por falta de tiempo debido al trabajo o por falta de conocimiento suficiente.

Por otro lado, el estudio realizado por (Cano-Hila \& Argemí-Baldich, 2021), recalca la influencia del capital cultural y educativo de los padres en la educación de sus hijos, sobre todo en un entorno virtual, donde es necesario que quienes cumplen el rol de complementar y reforzar el proceso de aprendizaje del estudiante tengan por lo menos un conocimiento básico de los contenidos de estudio, así como destrezas para el manejo de recursos tecnológicos. Este aspecto contrasta con los resultados obtenidos en nuestro estudio ya que la mayor parte de padres de familia no tienen ningún nivel educativo, o en el mejor de los casos solo han cursado la primaria, esto hace que el grado de comprensión de las tareas escolares sea muy limitado y por ende se vea afectado el desempeño académico de los estudiantes que sí requieren de un acompañamiento en casa.

\section{PROPUESTA}

Considerando los resultados obtenidos, mismos que muestran que a pesar de que existe el compromiso de los padres de familia por apoyar el proceso educativo de sus hijos, aspectos como la falta de tiempo y el desconocimiento de los contenidos y del manejo de recursos tecnológicos son las principales causas de la falta de acompañamiento efectivo a las tareas escolares. Como respuesta a esta problemática se propone la elaboración y 
aplicación del plan de actuación efectiva de la familia en el aprendizaje virtual, con el fin de promover el involucramiento familiar activo para mejorar el desempeño académico del estudiante. El plan consta de seis fases que se sintetizan a continuación en la figura 1.

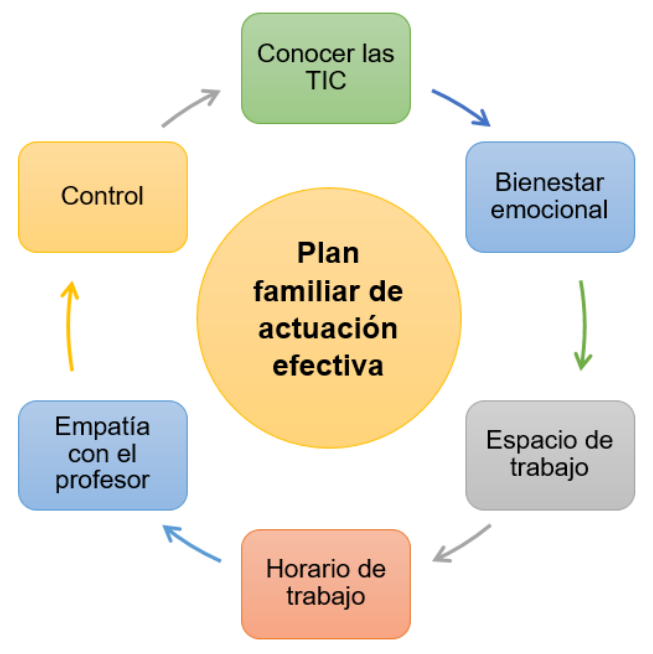

Figura 1. Plan de familiar de actuación efectiva. Elaboración: Los autores.

Conocer la TIC: crear un club de padres de familia, con el apoyo de los docentes tutores de cada paralelo se formarán grupos de WhatsApp en los que se irán compartiendo permanentemente instructivos, videos explicativos o tutoriales muy claros y concretos sobre el manejo de los diferentes recursos tecnológicos. De la misma forma se motivará los mismos estudiantes para que refuercen las habilidades digitales de sus padres. También se organizarán charlas breves y periódicas en las que el cada tutor apoyará en la explicación de ciertos temas complejos de comprender.

Bienestar emocional en el hogar: es fundamental que en el hogar se establezca un ambiente emocionalmente estable donde todos los miembros se sientan cómodos y tengan la suficiente confianza para expresar con claridad sus emociones y necesidades. Es importante también fomentar los espacios de diálogo para evitar confrontaciones, pero sobre todo para motivar constantemente al estudiante para que continúe estudiando y así evitar la deserción escolar. 
Establecer un espacio de trabajo adecuado: la familia debe establecer una zona específica destinada para el trabajo del estudiante, de preferencia bien iluminada y cómoda donde se organice todos los materiales y útiles escolares para que el estudiante se sienta gustoso de realizar sus tareas. De ser posible se deberá evitar distractores cercanos para facilitar la concentración y atención. Así mismo, el padre de familia consensuará con su hijo el aseo y orden permanentes de este espacio.

Horario de trabajo: es importante que se establezca un horario para el estudio diario con el fin de lograr una distribución del tiempo de trabajo serio y responsable. Este horario deberá contemplar también tiempos adecuados para el descanso y la ingesta de alimentos. Se debe organizar una agenda en la cual el estudiante vaya anotando las diferentes fechas para entrega de tareas, lecciones, reuniones o cualquier otra actividad planificada por el docente. De igual manera es recomendable que el padre de familia distribuya correctamente su tiempo entre sus labores diarias, destinando como mínimo 30 minutos diarios para el control de tareas de su hijo.

Empatía con el profesor: el padre de familia deberá considerar y valorar los esfuerzos que los docentes hacen por la educación de su hijo, por lo tanto, deberá estar atento a los comunicados o invitaciones a eventos o reuniones, tanto virtuales como presenciales, debe ser consiente que de que es parte fundamental del aprendizaje de su hijo. Es preciso también mantener una comunicación constante, fluida y sincera con el docente, de tal forma que siempre se mantenga al tanto del progreso académico de su representado.

Control eficiente de las actividades: es fundamental el compromiso del padre de familia con el proceso de aprendizaje, para lo cual es necesario que dedique tiempo de calidad a su hijo, diariamente deberá aprovechar el tiempo que le quede después de sus labores al control y revisión de las actividades educativas. Deberá tener presente también el control del uso adecuado de los recursos tecnológicos y dispositivos, evitando el uso excesivo de los mismos y monitoreando las aplicaciones, juegos, sitios web que visita, entre otros. 


\section{CONCLUSIONES}

La pandemia por COVID-19 ha puesto en evidencia los grandes retos que el sistema educativo debe superar para garantizar el derecho a la educación a todos por igual, para ello se han implementado una serie de adaptaciones buscando la continuidad del proceso académico desde casa. En este marco y a pesar de las grandes limitaciones, el rol de la familia sigue siendo fundamental, puesto que los comportamientos de los padres y el ambiente del hogar influyen en el resultado de aprendizaje de los estudiantes. Sin embargo, con la nueva modalidad de estudios han emergido nuevas necesidades que no todas las familias están en capacidad de solventar, es evidente que las diferencias en el acceso a recursos tecnológicos y de conectividad son muy marcadas, por ello es crucial que la escuela sea empática y se adapte a las características diversas de quienes aprenden, efectivizando estrategias conjuntas que permitan involucrar mejor a los padres con el fin de mejorar el rendimiento escolar.

Aplicar una metodología con un enfoque cuantitativo y cualitativo permite tener una visión más holística de la realidad investigada brindando una información más profunda y completa al obtener tanto, datos predictivos como proyecciones y conocimientos explicativos o contextuales. En cuanto a la aplicación de la encuesta mediante un formulario de Microsoft Forms, en cierto modo fue muy ventajoso por la rapidez de obtener información, sin embargo, se encontró una realidad diferente debido a que muchos de los encuestados no contaban con los recursos suficientes para completar en línea el cuestionario, por lo que fue necesario optar por llamadas telefónicas para cubrir todo el campo muestral.

Los resultados obtenidos en la investigación demostraron que existe un grado de involucramiento aceptable de la familia en el proceso de aprendizaje de sus hijos, sin embargo, se identificaron ciertas limitantes que impiden un acompañamiento más efectivo, siendo la falta de tiempo, el desconocimiento de contenidos, así como del manejo de recursos tecnológicos los más significativos. 
Al momento analizar las características socioculturales de la población investigada se identificó que gran parte de los padres de familia han cursado solo la primaria, otro grupo considerable no tiene ningún nivel académico, esto incide de forma significativa en el grado de comprensión de las tareas, puesto que muchos entienden algo y otro sector importante no las comprende nada. Por otro lado, se identificó que, si bien más de la mitad de familias disponen de acceso una red de Internet fijo, un gran porcentaje solo tienen un teléfono inteligente como recurso para recibir clases y realizar las tareas escolares.

La propuesta de un plan familiar de actuación efectiva permitirá que la familia organice un espacio de estudio, que gestione adecuadamente su tiempo en función de sus necesidades, priorizando el control y acompañamiento a las actividades educativas en un ambiente armónico que garantice el bienestar emocional, motivando el compromiso del representante de mantener una comunicación permanente, fluida y sincera con el docente, quien apoyará su alfabetización informacional aprovechando los recursos tecnológicos disponibles.

\section{FINANCIAMIENTO}

No monetario.

\section{AGRADECIMIENTO}

A la Jefatura de Posgrados de la Universidad Católica de Cuenca por permitir el desarrollo y fomento de la investigación. 
Revista Arbitrada Interdisciplinaria KOINONIA

Año VI. Vol VI. N³. Edición Especial: Educación II. 2021

Hecho el depósito de Ley: FA2016000010 ISSN: $2542-3088$

FUNDACIÓN KOINONIA (F.K). Santa Ana de Coro. Venezuela.

José Antonio Guaman-Duy; Sergio Constantino Ochoa-Encalada

\section{REFERENCIAS CONSULTADAS}

Alfonso, I. (2003). Elementos conceptuales básicos del proceso de enseñanzaaprendizaje. [Basic conceptual elements of the teaching-learning process]. ACIMED, 11(6).

Cano-Hila, A., \& Argemí-Baldich, R. (2021). Early Childhood and Lockdown: The Challenge of Building a Virtual Mutual Support Network between Children, Families and School for Sustainable Education and Increasing Their WellBeing. Sustainability. MDPI, Open Access Journal, 13(7), 1-15. https://doi.org/10.3390/su13073654

CEPAL-UNESCO. (2020). La educación en tiempos de la pandemia de COVID-19. [Education in times of the COVID-19 pandemic]. http://hdl.handle.net/11362/45904

Enríquez, L., \& Hernández, M. (2021). Alumnos en pandemia: una mirada desde el aprendizaje autónomo. [Students in a pandemic: a view from autonomous learning]. Revista Digital Universitaria, 22(2).

Formichella, M. M., \& Krüger, N. (2020). Pandemia y brechas educativas: Reflexiones desde la Economía. [Pandemic and educational gaps: Reflections from the Economy]. https://ri.conicet.gov.ar/handle/11336/109085

Hernández, R., Fernández, C., \& Baptista, P. (2014). Metodología de la investigación. [Investigation methodology] (Sexta). McGRAW-HILL / INTERAMERICANA EDITORES, S.A. DE C.V. https://n9.cl/2i4

Instituto Nacional de Estadisticas y Censo INEC. (2018). Tecnologías de la Información y Comunicación. Encuesta Multipropósito-TIC 2018. [Information and Communication Technologies. Multipurpose-ICT Survey 2018].

Méndez, J. \& Jaimes, L. (2018). Clima Social familiar e impacto en el rendimiento académico de los estudiantes [Family social climate and impact on the academic performance of students]. Perspectivas, 3(1). 24-43.

Ministerio de Educación. (2020a). Plan Educativo: aprendamos juntos en casa. [Educational Plan: let's learn together at home]. https://n9.cl/q079e

Ministerio de Educación. (2020b). Plan Educativo: aprendamos juntos en casa. Lineamientos Ámbito Pedagógico Curricular. [Educational Plan: let's learn together at home. Curricular Pedagogical Area Guidelines]. https://n9.cl/fc0zp 
Revista Arbitrada Interdisciplinaria KOINONIA

Año VI. Vol VI. N³. Edición Especial: Educación II. 2021

Hecho el depósito de Ley: FA2016000010 ISSN: 2542-3088

FUNDACIÓN KOINONIA (F.K). Santa Ana de Coro. Venezuela.

Muñoz-Moreno, J. L., \& Lluch-Molins, L. (2020). Educación y Covid-19: Colaboración de las Familias y Tareas Escolares [Education and Covid-19: Collaboration of Families and School Tasks]. Revista Internacional De Educación Para La Justicia Social, 9(3).

Oliveira, C., Oliveira, J., \& Azevedo, G. (2021). Parceria entre escola e família no desenvolvimento do aluno durante a a pandemia de Covid 19 [Partnership between school and family in student development during the Covid pandemic 19]. Revista de Estudos Em Educação, 7(1), 70-86.

Pire-Rojas, A. (2020). Instrumento para evaluar la relación escuela-familia en el proceso educativo virtual provocado por la Covid-19 [Instrument to evaluate the schoolfamily relationship in the virtual educational process caused by Covid19]. Conrado, 16(77), 298-306.

Razeto-Pavez, A. (2016). Estrategias para promover la participación de los padres en la educación de sus hijos: el potencial de la visita domiciliaria [Strategies to promote the participation of parents in the education of their children: the potential of home visit]. Estudios pedagógicos (Valdivia), 42(2), 449 462. https://dx.doi.org/10.4067/S0718-07052016000200026

Torrano, F, Fuentes, J, \& Soria, M. (2017). Aprendizaje autorregulado: estado de la cuestión y retos psicopedagógicos [Self-regulated learning: state of the issue and psycho-pedagogical challenges]. Perfiles educativos, 39(156), 160-173.

Vohlonen, A. (2020). COVID-19: Cómo asegurar el aprendizaje de los niños sin acceso a Internet. [COVID-19: How to ensure the learning of children without Internet access]. https://n9.cl/ya57

C2021 por los autores. Este artículo es de acceso abierto y distribuido según los términos y condiciones de la licencia Creative Commons Atribución-NoComercial-Compartirlgual 4.0 Internacional (CC BY-NC-SA 4.0)

(https://creativecommons.org/licenses/by-nc-sa/4.0/). 